Pacific Journal of Mathematic 


\title{
SOME CLASSES OF RINGS WITH INVOLUTION SATISFYING THE STANDARD POLYNOMIAL OF DEGREE 4
}

\author{
TAW-PIN LIM
}

The identities valid in the ring of real quaternions are defined in more general classes of rings with involution. With one exception, these classes of rings satisfy the standard polynomial of degree 4 and form a chain under inclusion. There are examples which show that these inclusions are proper. An example of an exterior algebra shows that a ring with involution whose symmetric elements commute does not necessarily satisfy the standard polynomial of degree 4 .

We assume that a ring has a unit although some of the proofs which follow do not require the existence of a unit in general.

Throughout the paper, $R$ will be a ring equipped with an involution *, i.e., a map $R \rightarrow R$ such that for all $x, y \in R,(x+y)^{*}=$ $x^{*}+y^{*},(x y)^{*}=y^{*} x^{*}$ and $x^{* *}=x$.

The sets $S$ and $K$ of symmetric and skew-symmetric elements of $R$ consist respectively of elements $x$ of $R$ such that $x^{*}=x$ and $x^{*}=-x$.

The trace and norm of an element $x$ in $R$ are respectively $T(x)=$ $x+x^{*}$ and $N(x)=x x^{*}$.

As usual, $[x, y]=x y-y x$ denotes the commutator, i.e., the standard polynomial of degree 2 of $x, y \in R$ and the symbol $Z$ denotes the center of $R$.

We shall require that a ring $R$ be subject to certain identities, all of which are valid in the case of real quaternions. In other words, we are extending the properties of real quaternions to more general classes of rings.

$R$ will be called a scalar product ring if for all $x, y \in R$,

$$
T(x y)=T(y x) \text {. }
$$

This definition follows from Dyson [1].

$R$ is called a normal ring if for all $x \in R, x x^{*}=x^{*} x$.

$R$ is called a central trace ring if for all $x \in R, T(x) \in Z$.

$R$ is called a central norm ring if for all $x \in R, N(x) \in Z$.

$R$ is called a central symmetric ring if the symmetric elements of $R$ are central i.e., $S \subset Z$.

$R$ is called a commuting symmetric ring if the symmetric elements of $R$ commute. 
Except for commuting symmetric rings, all the above classes of rings satisfy $S_{4}$, the standard polynomial of degree 4. A commuting symmetric ring does not necessarily satisfy $S_{4}$ even though its symmetric elements satisfy a polynomial of degree 2.

Clearly, a central symmetric ring is a central trace ring; a 2torsion free central trace ring is a central symmetric ring.

We shall first study scalar product rings.

From (1) we see that a scalar product ring satisfies

$$
[x, y]^{*}=-[x, y]
$$

or equivalently,

$$
\left[x^{*}, y^{*}\right]=[x, y] .
$$

A scalar product ring satisfies

$$
T([x, y] z)=T(x[y, z])
$$

because $T$ is additive and $T(y \cdot x z)=T(x z \cdot y)$. In particular, (4) implies $T([x, y] y)=0$ and hence

$$
[x, y] y=y^{*}[x, y] .
$$

THeOREM 1. A sacalar product ring $R$ stisfies the standard polynomial of degree 4 .

Proof. Let $a, b, c, d$ denote any four elements in $R$. The standard polynomial of degree 4 can be written in terms of commutators as follows:

$$
\begin{aligned}
S_{4}[a, b, c, d]= & {[a, b][c, d]+[b, c][a, d]+[c, a][b, d] } \\
& +[c, d][a, b]+[a, d][b, c]+[b, d][c, a] .
\end{aligned}
$$

By identities (2), (4) and the Jacobi identity respectively,

$$
\begin{aligned}
S_{4}[a, b, c, d] & =T([a, b][c, d])+T([b, c][a, d])+T([c, a][b, d]) \\
& =T([[a, b], c] d+[[b, c], a] d+[[c, a], b] d) \\
& =0 .
\end{aligned}
$$

As shown in [5], a normal ring is a scalar product ring and hence satisfies $S_{4}$. On the other hand, a 2-torsion free scalar product ring is a normal ring. In [5], there is an example of a scalar product ring which is neither 2-torsion free nor normal.

THEOREM 2. A central trace ring is a normal ring. Conversely, a normal ring with $2=0$ is a central trace ring. 
Proof. If $R$ is a central trace ring then for all $x \in R, x T(x)=$ $T(x) x$ and hence $x x^{*}=x^{*} x$.

Conversely, if $R$ is a normal ring with $2=0$ then $x x^{*}+x^{*} x=$ 0 for all $x \in R$. We have for all $x, y \in R$,

$$
\begin{aligned}
0 & =\left[x, y y^{*}+y^{*} y\right] \\
& =y\left[x, y^{*}\right]+[x, y] y^{*}+y^{*}[x, y]+\left[x, y^{*}\right] y \\
& =\left[x, y^{*}\right] y^{*}+[x, y] y^{*}+[x, y] y+\left[x, y^{*}\right] y, \quad \text { by }(5) \\
& =\left[x, y^{*}+y\right]\left(y^{*}+y\right) \\
& =[x, t] t \quad \text { where } t=y^{*}+y \\
& =t[x, t], \quad \text { by }(5) .
\end{aligned}
$$

The last two equalities imply

$$
x t^{2}=t x t=t^{2} x
$$

that is, $t^{2} \in Z$. Moreover,

$$
x t^{3}=t^{2} \cdot x t=t \cdot t x t=t \cdot t^{2} x=t^{3} x
$$

and hence $t^{3} \in Z$.

As $(1+t)^{3}=1+t+t^{2}+t^{3} \in Z$, we have $t \in Z$. Hence, $R$ is a central trace ring.

A normal ring is not a central trace ring in general since we have the following:

EXAMPLe 1 . Let $F$ be a field of char $\neq 2$ and $R$ be the $F$-algebra of triangular matrices of the form:

$$
x=\left[\begin{array}{rrrr}
a & b & c & d \\
& a & 0 & c \\
& & a & -b \\
& & & a
\end{array}\right]
$$

with

$$
x^{*}=\left[\begin{array}{rrrr}
a & b & c & -d \\
& a & 0 & c \\
& & a & -b \\
& & & a
\end{array}\right] .
$$

It is easy to verify that $*$ is an involution on $R$ and that $R$ is a normal ring. Clearly, $R$ is not a central trace ring.

Theorem 3. $R$ is a central norm ring if and only if $R$ is a central trace ring. 
Proof. If $R$ is a central norm ring then for all $x \in R$ we have $(1+x)(1+x)^{*} \in Z$ which implies $x+x^{*} \in Z$.

Conversely, if $R$ is a central trace ring and hence a scalar product ring, we have for all $x, y \in R$,

$$
\begin{aligned}
0 & =\left[x, y+y^{*}\right] y \\
& =[x, y] y+\left[x, y^{*}\right] y \\
& =y^{*}[x, y]+\left[x, y^{*}\right] y, \quad \text { by } \\
& =\left[x, y^{*} y\right] .
\end{aligned}
$$

Hence $y^{*} y \in Z$ for all $y \in R$ and so $R$ is a central norm ring.

THEOREM 4. A 2-torsion free scalar product ring $R$ with no nonzero central nilpotent elements is a central symmetric ring.

Proof. For all $a \in S, x \in K$, we have by (3) that $[a, x]=\left[a^{*}, x^{*}\right]=$ $-[a, x]$. Since $R$ is 2 -torsion free, $[a, x]=0$; that is, $S$ and $K$ commute elementwise. Consequently, $a x=x a=-(a x)^{*} \in K$.

Hence for all $a, b \in S, x \in K$, we have $b a x=a x b=a b x$ which yields $[a, b] x=0$. Similarly, $x[a, b]=0$. From $(2),[a, b] \in K$ and it follows that $[a, b]^{2}=0$.

Moreover for all $r \in R, 2 r[a, b]=\left(r+r^{*}+r-r^{*}\right)[a, b]=$ $T(r)[a, b]=[a, b] T(r)=2[a, b] r$. Therefore $[a, b] \in Z$.

As $[a, b]$ is central and nilpotent, we have by hypothesis that $[a, b]=0$ for all $a, b \in S$, i.e., $S$ is commutative.

Therefore $2[a, r]=\left[a, r+r^{*}+r-r^{*}\right]=0$ for all $a \in S, r \in R$ which implies $[a, r]=0$. That is, $R$ is a central symmetric ring.

Example 1 also shows that a 2-torsion free scalar product ring needs not be a central symmetric ring in general.

A central symmetric ring is of course a commuting symmetric ring. On the other hand, we have the following converse:

THEOREM 5. A semiprime commuting symmetric ring $R$ is a central symmetric ring.

Proof. Let $a \in S$ and $x \in R$. Since $\left[a, x+x^{*}\right]=0$, we have $[a, x]=-\left[a, x^{*}\right]=[a, x]^{*}$ and hence $[a, x] \in S$. Since $0=\left[a, x x^{*}\right]=$ $x\left[a, x^{*}\right]+[a, x] x^{*}$, we have $x[a, x]=-x\left[a, x^{*}\right]=[a, x] x^{*}=(x[a, x])^{*}$ and hence $x[a, x] \in S$.

Therefore $a \cdot x[a, x]=x[a, x] \cdot a=x a[a, x]$ which yields $[a, x]^{2}=0$ for all $a \in S, x \in R$.

For $a \in S$, suppose there exists $x \in R$ such that $b=[a, x] \neq 0$. Then $b^{2}=0$ and $[b, y]^{2}=0$ for all $y \in R$ from which we get $(b y)^{3}=$ $b(y b)^{2} y=b[b, y]^{2} y=0$. Hence $b R$ is a right ideal of $R$ in which the 
cube of every element is 0. By Lemma 1.1 in [2], $R$ would have a nonzero nilpotent ideal which is impossible for a semiprime ring. Therefore $[a, x]=0$ for all $a \in S$ and $x \in R$, i.e., $R$ is a central symmetric ring.

The above theorem has also been proved by Herstein in a different context in [3, pp. 59-61].

A commuting symmetric ring does not necessarily satisfy $S_{4}$ as the following example shows:

EXAMPLE 2. Let $F$ be a field of characteristic zero and $V$ be a $F$-vector space of dimension 4 . Let $R=\Lambda V=\bigoplus_{n=0}^{\infty} \bigwedge^{n} V$ be the exterior algebra over $V$.

To simplify the notations, we write $x y$, instead of the usual $x \wedge y$, as the ring multiplication in $R$.

Since $\Lambda^{n} V=0$ for all $n>4$, we have

$$
R=F \oplus V \oplus \Lambda^{2} V \oplus \Lambda^{3} V \oplus \Lambda^{4} V
$$

The additive homomorphism ${ }^{*}: R \rightarrow R$ defined by

$$
x^{*}=x_{0}-x_{1}-x_{2}+x_{3}+x_{4}
$$

for

$$
x=x_{0}+x_{1}+x_{2}+x_{3}+x_{4}
$$

where

$$
x_{i} \in \Lambda^{i} V, i=0,1, \cdots, 4 ;
$$

is an involution on $R$.

Indeed, if $x=x_{0}+x_{1}+x_{2}+x_{3}+x_{4}, y=y_{0}+y_{1}+y_{2}+y_{3}+y_{4}$ where $x_{i}, y_{i} \in \Lambda^{i} V, i=0,1, \cdots, 4$ then

$$
\begin{aligned}
x y= & x_{0} y_{0}+\left(x_{0} y_{1}+x_{1} y_{0}\right)+\left(x_{0} y_{2}+x_{1} y_{1}+x_{2} y_{0}\right) \\
& +\left(x_{0} y_{3}+x_{1} y_{2}+x_{2} y_{1}+x_{3} y_{0}\right)+\left(x_{0} y_{4}+x_{1} y_{3}+x_{2} y_{2}+x_{3} y_{1}+x_{4} y_{0}\right)
\end{aligned}
$$

and

$$
\begin{aligned}
(x y)^{*}= & x_{0} y_{0}-\left(x_{0} y_{1}+x_{1} y_{0}\right)-\left(x_{0} y_{2}+x_{1} y_{1}+x_{2} y_{0}\right) \\
& +\left(x_{0} y_{3}+x_{1} y_{2}+x_{2} y_{1}+x_{3} y_{0}\right)+\left(x_{0} y_{4}+x_{1} y_{3}+x_{2} y_{2}+x_{3} y_{1}+x_{4} y_{0}\right) \\
= & y_{0} x_{0}-\left(y_{1} x_{0}+y_{0} x_{1}\right)-\left(y_{2} x_{0}-y_{1} x_{1}+y_{0} x_{2}\right) \\
& +\left(y_{3} x_{0}+y_{2} x_{1}+y_{1} x_{2}+y_{0} x_{3}\right)+\left(y_{4} x_{0}-y_{3} x_{1}+y_{2} x_{2}-y_{1} x_{3}+y_{0} x_{4}\right) \\
= & \left(y_{0}-y_{1}-y_{2}+y_{3}+y_{4}\right)\left(x_{0}-x_{1}-x_{2}+x_{3}+x_{4}\right) \\
= & y^{*} x^{*} .
\end{aligned}
$$

It can easily be seen that

$$
\begin{aligned}
& S=F \oplus \Lambda^{3} V \oplus \Lambda^{4} V \\
& K=V \oplus \Lambda^{2} V
\end{aligned}
$$


and that $S$ is commutative. Hence $R$ is a commuting symmetric ring.

$R$ does not satisfy $S_{4}$ because if $a, b, c, d$ is a basis of $V$ over $F$, then since $[a, b]=a \wedge b-b \wedge a=2(a \wedge b)$, it follows from (6) that

$$
S_{4}[a, b, c, d]=24(a \wedge b \wedge c \wedge d) \neq 0 .
$$

The following example is a commuting symmetric ring which satisfies $S_{4}$ but is not a scalar product ring.

EXAMPLE 3. Let $C$ be the field of complex numbers and $R$ be the $C$-algebra of $2 \times 2$ matrices of the form:

$$
\left[\begin{array}{ll}
x & y \\
0 & \bar{x}
\end{array}\right], \quad x, y \in C \text {. }
$$

The map ${ }^{*}$ defined by

$$
\left[\begin{array}{ll}
x & y \\
0 & \bar{x}
\end{array}\right]^{*}=\left[\begin{array}{ll}
\bar{x} & y \\
0 & x
\end{array}\right]
$$

is an involution on $R$. It can be verified that the symmetric elements of $R$ commute and that $R$ satisfies $S_{4}$. However, $R$ is not a scalar product ring since for

$$
a=\left[\begin{array}{rr}
i & 0 \\
0 & -i
\end{array}\right], \quad b=\left[\begin{array}{ll}
0 & 1 \\
0 & 0
\end{array}\right],
$$

we have $T(a b) \neq T(b a)$.

REMARKS. This paper is an improvement on a part of the author's $\mathrm{Ph}$. D. thesis written under the supervision of Professor D. Ž. Djoković at the University of Waterloo, Ontario, Canada. The author also wishes to thank the referee of this paper for his helpful suggestions which result in the presentation of Example 2.

\section{REFERENCES}

1. F. J. Dyson, Quaternion determinants, Helvetica Physica Acta, 45 (1972), 289-302.

2. I. N. Herstein, Topics in Ring Theory, Univ. of Chicago Press, Chicago, 1969.

3. _- Rings with Involution, Univ. of Chicago Press, Chicago, 1976.

4. T. P. Lim, Determinants over a Class of Rings with Involution, $\mathrm{Ph}$. D. thesis, Univ. of Waterloo, Canada, 1976.

5. - Conjugacy of elements in a normal ring, Canad. Math. Bull., Vol. 20 (1), $1977,113-115$.

Received June 1, 1978 and in revised form August 2, 1979. 


\section{PACIFIC JOURNAL OF MATHEMATICS}

\section{EDITORS}

DONALD BABBITT (Managing Editor)

University of California

Los Angeles, California 90024

HUgo RossI

University of Utah

Salt Lake City, UT 84112

C. C. MOORE and ANDREW OGG

University of California

Berkeley, CA 94720

\section{J. DUGUNDJI}

Department of Mathematics University of Southern California Los Angeles, California 90007

R. Finn and J. Milgram Stanford University Stanford, California 94305

\section{ASSOCIATE EDITORS}

E. F. BECKENBACH

B. H. NEUMANN

F. WOLF

K. YoshidA

\section{SUPPORTING INSTITUTIONS}

UNIVERSITY OF BRITISH COLUMBIA CALIFORNIA INSTITUTE OF TECHNOLOGY UNIVERSITY OF CALIFORNIA MONTANA STATE UNIVERSITY UNIVERSITY OF NEVADA, RENO NEW MEXICO STATE UNIVERSITY OREGON STATE UNIVERSITY UNIVERSITY OF OREGON
UNIVERSITY OF SOUTHERN CALIFORNIA STANFORD UNIVERSITY UNIVERSITY OF HAWAII UNIVERSITY OF TOKYO UNIVERSITY OF UTAH WASHINGTON STATE UNIVERSITY UNIVERSITY OF WASHINGTON 


\section{Pacific Journal of Mathematics}

Vol. 85, No. $1 \quad$ September, 1979

Ralph Alexander, Metric averaging in Euclidean and Hilbert spaces...... 1

B. Aupetit, Une généralisation du théorème de Gleason-Kahane-Żelazko

pour les algèbres de Banach..............................

Lung O. Chung, Jiang Luh and Anthony N. Richoux, Derivations and

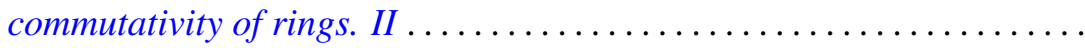

Lynn Harry Erbe, Integral comparison theorems for third order linear

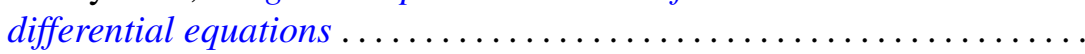

Robert William Gilmer, Jr. and Raymond Heitmann, The group of units of a

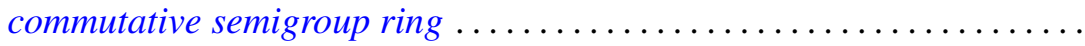

George Grätzer, Craig Robert Platt and George William Sands, Embedding lattices into lattices of ideals ...........................

Raymond D. Holmes and Anthony Charles Thompson, $n$-dimensional area and content in Minkowski spaces ....................... 77

Harvey Bayard Keynes and M. Sears, Modelling expansion in real flows....

Taw Pin Lim, Some classes of rings with involution satisfying the standard

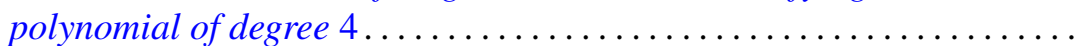

Garr S. Lystad and Albert Robert Stralka, Semilattices having bialgebraic congruence lattices ................................... 131

Theodore Mitchell, Invariant means and analytic actions . . . . . . . . . 145

Daniel M. Oberlin, Translation-invariant operators of weak type ........ 155

Raymond Moos Redheffer and Wolfgang V. Walter, Inequalities involving

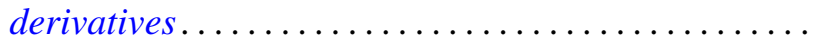

Eric Schechter, Stability conditions for nonlinear products and semigroups ................................

Jan Søreng, Symmetric shift registers ........................ 201

Toshiji Terada, On spaces whose Stone-Čech compactification is $\mathrm{Oz} \ldots \ldots .231$

Richard Vrem, Harmonic analysis on compact hypergroups ... 